\title{
VARIATION IN TOOTH CROWN SIZE AND SHAPE ARE OUTCOMES OF THE COMPLEX ADAPTIVE SYSTEM ASSOCIATED WITH THE TOOTH NUMBER VARIATION OF HYPODONTIA
}

\author{
SADAF SASSANI ${ }^{1}$, DILAN PATEL ${ }^{1}$, MAURO FARELLA $^{2}$, MACIEJ HENNEBERG ${ }^{1}$, SARBIN RANJITKAR $^{1}$, \\ ROBIN YONG ${ }^{1}$, STEPHEN SWINDELLS ${ }^{2} \&$ ALAN H. BROOK ${ }^{1,3}$ \\ ${ }^{1}$ The University of Adelaide, the School of Dentistry, South Australia. \\ ${ }^{2}$ The University of Otago, the School of Dentistry, New Zeland. \\ ${ }^{3}$ Queen Mary University, Institute of Dentistry, London.
}

\begin{abstract}
The development of the dentition is a good model of general development; it has the general characteristics of a complex adaptive system. The developmental variation of hypodontia presents with a reduced number of teeth with several other phenotypic changes. The teeth formed are smaller in size, have different crown and root morphology and are delayed in development. The present study is a component of a multi-centre and multidisciplinary collaborative study to investigate hypodontia from genotype to phenotype. This study uses enhanced 3D-imaging techniques in order to increase the range of parameters of the phenotypic outcome: tooth size and tooth shape. The sample consists of orthodontic patients, 60 with hypodontia (30 males and 30 females), and 60 controls matched for age, sex and ethnicity. The material studied for these measurements are the dental models of each patient; these have been imaged with an Amann Girrbach Ceramill Map400 3D scanner. The 3D images produced were all taken by one operator and viewed on MeshLab. The accuracy of the measurements taken was determined through repeat measurements of the same images, undertaken to determine intra and interoperator reproducibility. This new system was validated by repeating these measurements using the standard 2D caliper technique. Ten repeat measurements were taken on ten models of the lower and upper premolar inter-cuspal distances. The average intra-operator reproducibility for the inter-cuspal distances when measuring the distance between the buccal and palatal cusp of the maxillary premolar was $0.20 \mathrm{~mm}$; the mandibular premolar was $0.32 \mathrm{~mm}$. The results for inter-operator reproducibility demonstrate an average difference of $0.24 \mathrm{~mm}$ for the maxillary premolar and $0.16 \mathrm{~mm}$ for the lower premolar. This novel method provides an increased range of measurements with good levels of accuracy. This study will go on to establish the variations on the 3D images between the hypodontia and the control group.

Keywords: 3D-Imaging, complex adaptive system, error, hypodontia, inter, intra, linear, measurement, reliability, reproducibility.
\end{abstract}

\section{INTRODUCTION}

This is one component of a multi-centre and multi-disciplinary study to investigate the complex relationship between the genotype and phenotype in hypodontia patients. The component reported here will compare the crown dimensions in patients with hypodontia to matched controls. Furthermore, as novel 3D measurement techniques are used it is necessary to determine the intra and inter-operator error to ascertain the degree of operator reliability; this paper will outline the procedure used to determine this.

\section{HYPODONTIA}

The majority of the population develops 20 primary teeth and 32 secondary teeth. When a specific tooth does not form this is known as tooth agenesis; the most frequently affected teeth are the third molars [1]. The dentition may be affected in varying numbers of missing teeth; hypodontia, which is the absence of one or up to five teeth, oligodontia, which involves six teeth or 
more and anodontia, which is the total absence of teeth [1]. Oligodontia and anodontia occur infrequently in a population and are commonly associated with syndromes such as ectodermal dysplasia [2]. However, hypodontia is one of the most commonly occurring human dental variations [3]. The remaining teeth present in an individual affected by hypodontia are smaller in dimensions, have different crown and root morphology and are delayed developmentally.

In biological development complex adaptive systems (CAS) refer to a dynamic process where lower level interactions produce higher level structure and phenomena [4]. Dental development has been shown to have the features of a CAS and can provide a good paradigm for general development as well as links with other autonomous complex adaptive systems such as fingerprint development [4-6]. Key characteristics and components of dental development include multidimensional, multi-level and multifactorial properties; the resulting phenotype is the product of multiple interactions between epigenetic, genetic and environmental factors [4-7]. Environmental changes within the developing dental CAS, such as increased intrauterine male hormones, have demonstrated phenotypic changes in tooth size, shape and dental arches [8,9].

\section{MATERIALS AND METHODS}

\subsection{Study sample}

The sample comprises 30 female and 30 male orthodontic patients from The University of Otago with mild-to-moderate hypodontia (one to five missing teeth) in their permanent dentition. This has been confirmed via an orthopantomograph. A corresponding group of 30 female and 30 male patients who have a complete dentition acts as the controls. The age range of the male hypodontia group was 10.2-21.0 years, the female hypodontia group was 10.8-19.3 years, the male control group was 10.1-17.3 years and the female control group was 10.6-19.2 years. The sample size was estimated by Brook et al. [10]; suggesting a comparison of two groups of twenty will give an $80 \%$ power to determine a size difference of 0.90 millimeters. The samples used to determine the intra- and inter-operator error were from ten additional patients that were a part of the control pool.

\subsection{Study materials}

The patients' dental casts were duplicated using polyvinyl siloxane (PVS) impression material at The University of Otago. The impressions were poured in die stone and trimmed. The dental casts were then scanned using an Amann Girrbach Ceramill Map400 by the same operator; this produced a 3D digital model in a STL file format.

\subsection{Ethical approval}

Ethical approval was granted by the Human Research Ethics Committee of The University of Adelaide; this work was deemed to be of negligible risk.

\subsection{D-Image analysis}

The 3D digital model, or images, produced from the dental casts were extremely detailed and data dense, in part because they were constructed from a twenty-micron resolution or less. The 3D models were then measured on MeshLab software for the premolar inter-cuspal distance. 


\subsection{Inter-cuspal distance}

The teeth selected for measurement were the upper second premolar on the right side, and the lower second premolar on the left side. The inter-cuspal distance was defined as the distance between the cusp tips (Fig. 1). Some lower premolars have additional cusp tips, in this instance it was decided to take the distance from the distal cusp tip.

\subsection{Intra- and inter-operator error}

The intra-operator error was determined by having the first operator take ten initial intercuspal measurements from the upper and lower second premolar. The first operator then took the same measurements from the same teeth eight weeks later and the differentials between the two sets of measurements were calculated.

The inter-operator error was determined by having a second operator take the same ten inter-cuspal measurements from the upper and lower second premolar, the differentials between the first and second operator were then calculated.

\section{RESULTS}

The results are presented in the following Tables 1-4. Tables 1 and 2 show the values the first operator measured on two separate occasions with the differentials (intra-operator), followed by the mean of the differentials, which were $0.20 \mathrm{~mm}$ and $0.32 \mathrm{~mm}$ for teeth the upper and lower second premolar, respectively. Tables 3 and 4 show the values the first operator and the second operator measured followed by the difference between them (inter-operator); the average for this was $0.09 \mathrm{~mm}$ and $0.58 \mathrm{~mm}$ for the upper and lower second premolar, respectively. Using SPSS software an intra-class correlation coefficient model (ICC), specifically a two-way random effects with absolute agreement, was applied to the values produced by the first and second operators to assess their reliability. Values produced for an ICC range from 0 to -1 , where 1 is a perfect correlation. The ICC for the first operator's intra-operator reliability was 0.818 for the upper second premolar and 0.852 for the lower second premolar. The ICC for the first operator's and the second operator's inter-operator reliability was 0.955 for the upper second premolar and 0.685 for the lower second premolar.

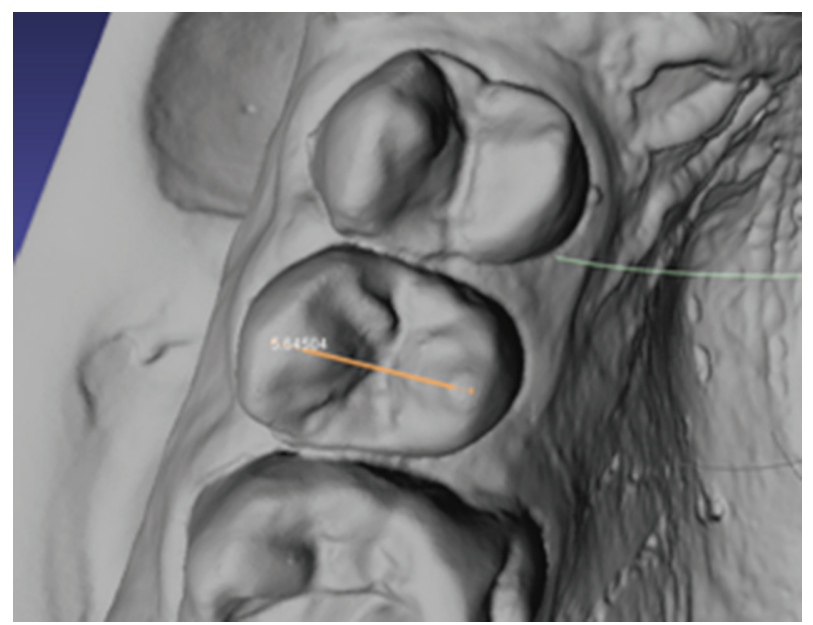

Figure 1: Premolar inter-cuspal distance measurement. 
Table 1: First operator intra-operator error differentials for the upper second premolar ( $\mathrm{mm})$.

First operator measurements of First operator measurements of the upper the upper second premolar second premolar eight weeks later

Differentials

\begin{tabular}{lll}
5.49148 & 5.72686 & 0.23538 \\
5.7727 & 5.5659 & 0.2068 \\
5.89043 & 5.61694 & 0.27349 \\
$\mathrm{n} / \mathrm{a}$ & $\mathrm{n} / \mathrm{a}$ & $\mathrm{n} / \mathrm{a}$ \\
6.2691 & 6.12684 & 0.14226 \\
$\mathrm{n} / \mathrm{a}$ & $\mathrm{n} / \mathrm{a}$ & $\mathrm{n} / \mathrm{a}$ \\
5.4855 & 5.35244 & 0.13306 \\
6.40191 & 6.20258 & 0.19933 \\
5.50001 & 5.72432 & 0.22431 \\
$\mathrm{n} / \mathrm{a}$ & $\mathrm{n} / \mathrm{a}$ & $\mathrm{n} / \mathrm{a}$ \\
\hline
\end{tabular}

Mean: 0.20209

Table 2: First operator intra-operator error differentials for the lower second premolar (mm).

First operator measurements First operator measurements of the of the lower second premolar upper second premolar eight weeks later Differentials

\begin{tabular}{lll}
\hline 5.96405 & 6.09306 & 0.12901 \\
4.08941 & 4.53681 & 0.4474 \\
5.87754 & 6.02671 & 0.14917 \\
$\mathrm{n} / \mathrm{a}$ & $\mathrm{n} / \mathrm{a}$ & $\mathrm{n} / \mathrm{a}$ \\
5.28945 & 5.47873 & 0.18928 \\
4.30977 & 3.26884 & 1.04093 \\
5.17975 & 5.07707 & 0.10268 \\
5.45517 & 5.22245 & 0.23272 \\
5.0966 & 4.85478 & 0.24182 \\
$\mathrm{n} / \mathrm{a}$ & $\mathrm{n} / \mathrm{a}$ & $\mathrm{n} / \mathrm{a}$ \\
\hline
\end{tabular}

Mean: 0.31662625

\section{DISCUSSION}

ICC values of above 0.75 are considered to indicate good reliability $[11,12]$. All the values for intra- and inter-operator reliability, except for the lower second premolar, were above this threshold and it suggests there is evidence of reliability. The reasons for the lower second premolar having decreased reliability likely stems from its morphology; it can have one or two lingual cusp tips and this could be open to interpretation by the operator. Some degree of variability between operators will always exist when operators choose anatomical points because of their interpretation of where that anatomical landmark is. Variability also exists between operators measuring with traditional calipers, plaster models, and where the points have been 
Table 3: First and second operator inter-operator error for the upper second premolar ( $\mathrm{mm}$ ).

\begin{tabular}{lll}
\hline $\begin{array}{l}\text { First operator upper second } \\
\text { premolar measurements }\end{array}$ & $\begin{array}{l}\text { Second operator upper second } \\
\text { premolar measurements }\end{array}$ & Differentials \\
\hline 5.49148 & 5.538 & 0.04652 \\
5.7727 & 5.709 & 0.0637 \\
5.89043 & 6.089 & 0.19857 \\
n/a & n/a & n/a \\
6.2691 & 6.34 & 0.0709 \\
n/a & n/a & n/a \\
5.4855 & 5.48 & 0.0055 \\
6.40191 & 6.233 & 0.16891 \\
5.50001 & 5.583 & 0.08299 \\
n/a & n/a & n/a \\
\hline
\end{tabular}

Mean: 0.091012857

Table 4: First and second operator inter-operator error for the lower second premolar ( $\mathrm{mm})$.

\begin{tabular}{lll}
\hline $\begin{array}{l}\text { First operator lower second } \\
\text { premolar measurements }\end{array}$ & $\begin{array}{l}\text { Second operator lower second } \\
\text { premolar measurements }\end{array}$ & Differentials \\
\hline 5.96405 & 5.63 & 0.33405 \\
4.08941 & 3.77 & 0.31941 \\
5.87754 & 5.377 & 0.50054 \\
$\mathrm{n} / \mathrm{a}$ & $\mathrm{n} / \mathrm{a}$ & $\mathrm{n} / \mathrm{a}$ \\
5.28945 & 5.127 & 0.16245 \\
4.30977 & 3.325 & 0.98477 \\
5.17975 & 3.969 & 1.21075 \\
5.45517 & 5.212 & 0.24317 \\
5.0966 & 4.195 & 0.9016 \\
$\mathrm{n} / \mathrm{a}$ & $\mathrm{n} / \mathrm{a}$ & $\mathrm{n} / \mathrm{a}$ \\
\hline
\end{tabular}

Mean: 0.5820925

marked for them to measure; simply due to the slight variation in the manual positioning of the calipers [13]. Previous studies have demonstrated that, while there is inter-operator error when measuring on a 3D model, this is still less than traditional plaster models and calipers [13-15]. Digital models may be more reliable than hand calipers because of the ability to zoom in and rotate the models in multiple planes [15].

\section{CONCLUSION}

The evidence from this study and the literature suggests that measurements taken on 3D digital models are more reliable than traditional caliper hand measurements. In the future if all dental models are stored as 3D-images, with a high quality and accurate resolution, the primary source of error amongst operators will stem from interpretation of anatomical 
landmarks. The initial study reported here provides a basis for the future detailed studies of the crown size and shape in these hypodontia patients and controls.

\section{ACKNOWLEDGEMENTS}

We are grateful for the participation of the patients in this study and we would also like to acknowledge the assistance of Dr. M Sassani, Dr. D Haag, and the work of the dental technicians at The University of Otago.

\section{REFERENCES}

[1] AlShahrani, I., Togoo, R.A. \& AlQarni, M.A., A review of hypodontia: classification, prevalence, etiology, associated anomalies, clinical implications and treatment options. World Journal of Dentistry, 4(2), pp. 117-125, 2013. https://doi.org/10.5005/jp-journals-10015-1216

[2] Werther, R. \& Rothenberger, F., Anodontia, a review of its etiology with presentation of a case. American Journal of Oral Surgery, 25, pp. 61-81, 1939. https://doi.org/10.1016/S0096-6347(39)90349-2

[3] Brook, A.H., Dental anomalies of number, form and size: their prevalence in British schoolchildren. Journal of the International Association of Dentisity for Children, 5(2), pp. 37-53, 1974.

[4] Brook, A.H., O’Donnell, M.B., Hone, A., Hart, E., Hughes, T.E., Smith, R.N. \& Townsend, G.C., General and craniofacial development are complex adaptive processes influenced by diversity. Australian Dental Journal, 59, pp. 13-22, 2014. https://doi.org/10.1111/adj.12158

[5] Taduran, R.J.O., Ranjitkar, S., Hughes, T., Townsend, G. \& Brook, A.H., Complex systems in human development: sexual dimorphism in teeth and fingerprints of Australian twins. International Journal of Design \& Nature and Ecodynamics, 11(4), pp. 676-685, 2016. https://doi.org/10.2495/DNE-V11-N4-676-685

[6] Brook, A., Koh, K. \& Toh, V., Influences in a biological complex adaptive system: environmental stress affects dental development in a group of Romano-Britons. International Journal of Design \& Nature and Ecodynamics, 11(1), pp. 33-40, 2016. https://doi.org/10.2495/DNE-V11-N1-33-40

[7] Koh, K., Toh, V., Brook O’Donnell, M., Ranjitkar, S. \& Brook, A., A complex adaptive system in which environmental stress affects gene expression during development. International Journal of Design \& Nature and Ecodynamics, 11(4), pp. 686-695, 2016. https://doi.org/10.2495/DNE-V11-N4-686-695

[8] Lam, F., Yong, R., Ranjitkar, S., Townsend, G.C. \& Brook, A.H., Agents within a developmental complex adaptive system: intrauterine male hormones influence human tooth size and shape. International Journal of Design \& Nature and Ecodynamics, 11(4), pp. 696-702, 2016.

https://doi.org/10.2495/DNE-V11-N4-696-702

[9] Patel, P., Yong, R., Ranjitkar, S., Townsend, G. \& Brook, A., Agents within a development complex adaptive system: intrauterine male hormones and dental arch size in humans. International Journal of Design \& Nature and Ecodynamics, 11(4), pp. 703-711, 2016.

https://doi.org/10.2495/DNE-V11-N4-703-711 
[10] Brook, A.H., Elcock, C., Al-Sharood, M.H., McKeown, H.F., Khalaf, K. \& Smith, R.N., Further studies of a model for the etiology of anomalies of tooth number and size in humans. Connective Tissue Research, 43(2-3), pp. 289-295, 2002. https://doi.org/10.1080/03008200290000718

[11] Cicchetti, D., Guidelines, criteria, and rules of thumb for evaluating normed and standardized assessment instruments in psychology. Psychological Assessment, 6(4), pp. 284-290, 1994.

https://doi.org/10.1037/1040-3590.6.4.284

[12] Harris, E.F. \& Smith, R.N., Accounting for measurement error: a critical but often overlooked process. Archives of Oral Biology, 54(1), pp. 107-117, 2008.

[13] Bell, A., Ayoub, A. \& Siebert, P., Assessment of the accuracy of a three- dimensional imaging system for archiving dental study models. Journal of Orthodontics, 30, pp. 219-223, 2003. https://doi.org/10.1093/ortho/30.3.219

[14] Moreira, D., Gribel, B., Torres, G., Vasconcelos, K., Freitas, D. \& Ambrosano, G., Reliability of measurements on virtual models obtained from scanning of impressions and conventional plaster models. Brazilian Journal of Oral Sciences, 13(4), pp. 297 302, 2014. https://doi.org/10.1590/1677-3225v13n4a11

[15] Stevens, D., Mir, C., Nebbe, B., Raboud, D., Heo, G. \& Major, P., Validity, reliability, and reproducibility of plaster vs digital study models: comparison of peer assessment rating and Bolton analysis and their constituent measurements. American Journal of Orthodontics and Dentofacial Orthopedics, 129, pp. 794-803, 2006.

https://doi.org/10.1016/j.ajodo.2004.08.023 